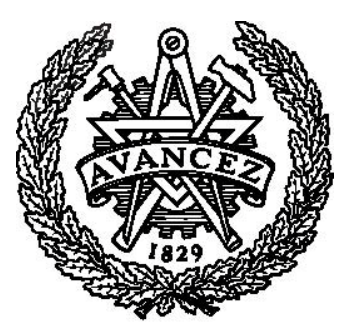

CHALMERS

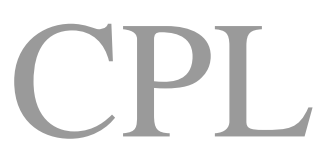

Chalmers Publication Library

Institutional Repository of Chalmers University of Technology

http://publications . lib. chalmers . se

This is an author produced version of a paper published in Energy Policy.

This paper has been peer-reviewed but does not include the final publisher proof-corrections or journal pagination.

Citation for the published paper:

Elin Svensson, Thore Berntsson, Ann-Brith Strömberg and Michael Patriksson

An optimization methodology for identifying robust process integration investments under uncertainty

Energy Policy, 2009, Vol. 37, Issue 2: pp 680-685

URL: http://dx.doi.org/10.1016/j.enpol.2008.10.023

Access to the published version may require subscription. Published with permission from:

Elsevier 


\title{
An optimization methodology for identifying robust process integration investments under uncertainty
}

\author{
Elin Svensson ${ }^{\mathrm{a}, *}$, Thore Berntsson ${ }^{\mathrm{a}}$, Ann-Brith Strömberg ${ }^{\mathrm{b}}$, Michael Patriksson ${ }^{\mathrm{c}}$ \\ ${ }^{a}$ Heat and Power Technology Group, Department of Energy and Environment, Chalmers University of \\ Technology, SE-412 96 Göteborg, Sweden. \\ ${ }^{b}$ Fraunhofer-Chalmers Research Centre for Industrial Mathematics, Chalmers Science Park, SE-412 88 \\ Gothenburg, Sweden. Presently at Department of Mathematical Sciences, Chalmers University of \\ Technology and Department of Mathematical Sciences, University of Gothenburg, SE-412 96 Göteborg, \\ Sweden. \\ ${ }^{c}$ Department of Mathematical Sciences, Chalmers University of Technology and Department of \\ Mathematical Sciences, University of Gothenburg, SE-412 96 Göteborg, Sweden.
}

\begin{abstract}
Uncertainties in future energy prices and policies strongly affect decisions on investments in process integration measures in industry. In this paper, we present a fivestep methodology for the identification of robust investment alternatives incorporating explicitly such uncertainties in the optimization model. Methods for optimization under uncertainty (or, stochastic programming) are thus combined with a deep understanding of process integration and process technology in order to achieve a framework for decision-making concerning the investment planning of process integration measures under uncertainty. The proposed methodology enables the optimization of investments in energy efficiency with respect to their net present value or an environmental objective. In particular, as a result of the optimization approach, complex investment alternatives, allowing for combinations of energy efficiency measures, can be analyzed. Uncertainties as well as time-dependent parameters, such as energy prices and policies, are modelled using a scenario-based approach, enabling the identification of robust investment solutions. The methodology is primarily an aid for decision-makers in industry, but it will also provide insight for policy-makers into how uncertainties regarding future price levels and policy instruments affect the decisions on investments in energy efficiency measures.
\end{abstract}

Keywords: stochastic programming, process integration, investment planning.

\section{Introduction}

\subsection{Background}

Several studies have shown that there are many opportunities for increasing the energy efficiency in industry by using process integration (IEA, 2007), but despite the fact that many of the suggested measures are shown to be profitable, few have actually been implemented. One of the main reasons seems to be that companies are not willing to take the risk associated with making such investment decisions because they lack good background information on their possible outcomes. The economically optimal choice of energy efficiency measures is dependent on, for example, future energy prices, policy

\footnotetext{
* Corresponding author. Tel.: +46 3177230 16; fax: + 4631821928.

E-mail address: el i n. svensson@hal mer s. se (Elin Svensson)
} 
instruments and the availability of new technologies, all of which are highly uncertain entities.

Although studies, for example in the pulp and paper industry (FRAM, 2005; KAM, 2003), show that many energy efficiency projects can become profitable, the conclusions from the studies are rarely clear on which measures to take, but rather give different advice for different scenarios. This is of course of little help when decisions have to be made before the outcome of uncertain parameters is known; picking the best decision for a high-price scenario could be the worst thing to do if ending up in the low-price scenario, and vice versa. A decision that is not the best one for any scenario could still be the best choice overall, because it acts as a hedge against future uncertainties, and thereby has a good enough profitability for every scenario. Such a solution, which is good for a variety of values of the uncertain parameters, is here said to be robust.

Uncertainties are especially important to consider in the optimization of strategic investments, when the decisions have to be made in the near future, but the investment will be affected by long-term variations and changes in energy market parameters.

In analyses of energy systems, one common way of dealing with uncertain parameters is to carry out a sensitivity analysis (Saltelli et al., 2004) to analyze the stability of the solution. Sensitivity analysis is a way to study how an optimal solution, that is, the optimal set of decisions, will change with changes in the input parameters. However, the optimal decisions to be made under uncertainty may never be revealed using this approach. Wallace (2000) posed the question: 'Is sensitivity analysis of any use?', and showed that it is far from being a good substitute for incorporating uncertainty directly into the optimization modelling. In short, sensitivity analysis will never reveal solutions for which flexibility has a cost, since flexibility is not an issue when each scenario is analyzed separately. When decisions have to be made before uncertainties are resolved, flexibility will, however, be of value, which might make it worth the extra cost. By formulating the optimization of investment decisions in a stochastic programming model, flexible solutions are considered, in addition to all of the solutions that would be revealed using sensitivity analysis.

\subsection{Aims and scope}

The aim of this work is to use existing mathematical modelling and methods for optimization under uncertainty (or, stochastic programming), and incorporate knowledge of process integration and process technology in order to achieve a better framework for decision-making regarding energy efficiency measures. The objective is to find the combination of investments that results in the highest net present value (NPV), which is expressed by the formula

$$
\mathrm{NPV}=-C_{0}+\sum_{t=1}^{T} \frac{C_{t}}{(1+r)^{t}}
$$

where $T$ is the economic lifetime (in years) of investments, $r$ is the discount rate, $C_{0}$ is the initial investment, and $C_{t}$ is the net cash flow (revenues minus costs) in year $t$. The net cash flow of the final year, $C_{T}$, is adjusted for the value remaining after the economic lifetime (the residual value). We have developed a multistage stochastic optimization model to improve the investment planning of energy efficiency measures under price and policy uncertainty. Here, instead of maximizing NPV for each scenario separately, the 
expected value of NPV over all scenarios is maximized. The initial investment is required to be the same for all scenarios since the first investment decision is taken before the outcome of the uncertain parameters is known. The objective function is hence to

$$
\text { maximize } E[N P V(x)]:=-C_{0}\left(x_{0}\right)+\sum_{s \in S} p_{s} \sum_{t=1}^{T} \frac{C_{t}\left(x_{0}, x_{s}, \omega_{s}\right)}{(1+r)^{t}},
$$

where $\mathrm{E}$ denotes expectation, $S$ denotes the set of all scenarios $s, p_{s}$ is the probability for scenario $s$ to occur, $x=\left(x_{0}, x_{s}\right)$ where $x_{s}$ is a vector of decision variables corresponding to scenario $s$ and representing, e.g., investment decisions or operating plans, and $x_{0}$ is the vector of decision variables associated with the initial investment and which is independent of $s$. Further, the initial investment $C_{0}$ is a piecewise linear function of the decision variables, $x_{0}$, and the net cash flow, $C_{t}$, in year $t$ is a function of the decisions, $x$, and the uncertainty parameters, $\omega_{s}$, for scenario $s$. By optimizing the objective function given by Eq. (2), we expect to find solutions that improve the investment planning of process integration measures, both since this methodology may produce solutions that are not found in a traditional investment analysis, and since it makes it possible to find solutions that are robust (Svensson et al., 2008b).

When incorporating uncertainty into investment decision modelling, the resulting model naturally becomes a stochastic integer programming model (see the paragraph below). This is the framework that our methodology is based on. However, something should be said about the real options approach that explains the underlying economic assumptions for such a framework, which in this application can be regarded as a special case of stochastic programming. While traditional economic decision rules are based on either reversibility of investments or 'now-or-never' statements, such assumptions are in many cases not valid in real situations. Investments can usually not be 'undone', but there is often a possibility of delaying them. When uncertainty is introduced, there might be a value of waiting if it leads to more relevant information being revealed and uncertainty thus being reduced. By investing immediately, this value would be lost. If there is a value of waiting, there is, in other words, an opportunity cost of investing. This opportunity cost is not accounted for by traditional investment rules (Dixit and Pindyck, 1994).

Multistage stochastic programming is one methodology for modelling the real options investment problem. The theory of stochastic linear programming is covered in, e.g., Birge and Loveaux (1997) and more recently in Ruszczyński and Shapiro (2003) and Kall and Mayer (2005). As for deterministic optimization, the introduction of integer or binary requirements on variable values makes the model considerably more time consuming to solve. The scenario tree modelling of the random variables further increases the size of the problem. However, the model will at least remain mixed-binary linear also with the introduction of uncertainties and will be possible to solve. Stochastic integer programming is described in, e.g., Louveaux and Schultz (2003) and Sen (2005).

In stochastic programming, it is acknowledged that decisions have to be made before uncertainties are resolved. This introduces at least two stages in the decision-making process. In this application, the first-stage decision is made on which initial investments should be carried out. As time goes on, the true values of the uncertain parameters such as energy prices, are revealed. Then, in the second stage there is a possibility of reacting to the outcome of these resolved uncertainties. For our application, the second-stage decision concerns the operating plans for the different technologies in which investments were made in the first stage, in order to achieve maximum revenues. Second-stage decisions also involve decisions on further investments to be made at that stage, as a reaction to the outcome of the uncertain parameters. This kind of model with two types of 
decisions where the second one is a reaction to the first, as well as on the realization of the uncertain parameters, is termed a recourse model. The division of decisions into two stages is one of the main differences of stochastic programming compared to deterministic optimization and sensitivity analysis.

Since investment decisions in industry must be based on economic profitability, the default objective is an economic measure. However, not only have environmental issues attracted increasing concern, but future $\mathrm{CO}_{2}$ emissions targets and policies are also strongly related to the uncertainties affecting these kinds of investments. Because of that, the model is adapted for analyzing $\mathrm{CO}_{2}$ emissions. In this way, the trade-off between economic and environmental objectives can be studied.

Also for policy-makers promoting energy-efficiency measures in industry, uncertainties are important to consider. Industries that invest in energy efficiency measures contribute to a reduction of $\mathrm{CO}_{2}$ emissions. A high $\mathrm{CO}_{2}$ emissions charge (in the form of a tax or tradable emission permit price) makes investments in $\mathrm{CO}_{2}$ emissions reductions more favourable. On the other hand, if the future charge level is unknown, there is a high uncertainty regarding the expected future cash flows. Therefore, investments might not be realized although they should be favourable, because of the higher risk and increased difficulty of how to analyze the different investment opportunities.

\subsection{Related work}

There are, to our knowledge, not yet any studies in which uncertainty about future energy market conditions is explicitly incorporated in the economic optimization of investments in process integraton or plant-wide energy efficiency. If uncertainty is accounted for it is by post-optimization sensitivity analysis, see e.g. Ådahl and Harvey (2007).

There are, however, several energy-related studies, which are presented below, which deal with decisions about investments and they all confirm the importance of accounting for uncertainty and timing.

Birge and Rosa (1996) incorporate investment uncertainty into optimization models to determine the optimal policy for investments in new energy technologies on a U.S. region scale. They show that simply by ignoring uncertainty, possibly $2 \%$ of overall economic activity may be lost. Investment decisions are, however, normally not taken at a national level. In our model the system is limited to what the decision-maker actually has economic control over, which typically is the energy system of one particular plant.

Blyth et al. (2007) investigate investment risks under uncertainty in a study of the timing of investments in power generation and, as in our model, they see the system from the point of view of a private company. Their main conclusion is that, in order for policymakers to promote low-carbon technologies, some long-term certainty about the future policy development should be provided. Yang et al. (2008) use the same methodology and conclude that uncertainty becomes more important to consider if the time between decision and policy event is short, because the value of waiting for better information increases. The effects of the uncertainties vary depending on technology and the way variations in carbon, fuel, and electricity prices are correlated. Fuss et al. (2008) studied the investment decision-making in the electricity sector under two types of uncertainty. Market uncertainty coming from fluctuations around a known trend was shown not to be of much concern. Uncertainty due to the absence of clear policy signals, however, was shown to lead to postponed investments in environmentally friendly technologies. They 
concluded that with a clear, long-term commitment plan, emissions abatement activities would start earlier.

Laurikka (2006) shows that, within an emissions trading scheme, a traditional investment decision rule concerning investments in IGCC (Integrated Gasification and Combined Cycle) technology may lead to biased results compared to an approach where uncertainties and real options are accounted for. Wickart and Madlener (2007) develop a model for the choice between combined heat and power production and heat-only production for an industrial firm. The model accounts for uncertainties in energy prices and determines the best technology choice as well as the timing of the investment. They suggest that for policy-making, the interrelationship between policies and energy price variability should be accounted for. Diederen et al. (2003) explain the gap between the expected and the observed adoption of energy-saving technologies in the Dutch horticultural sector by using a real options approach. They explained at least part of the observed gap to be due to uncertainties in energy prices.

A difference between our model and all of those mentioned above is that, in our case, the investment decisions affect each other in a very direct way, making it impossible to associate a cash flow to a specific investment. An example is the benefit from the investment in a turbine, which cannot be determined without knowing to what extent investments in steam savings have been carried out, and to what extent the acquired steam is used in the turbine. The benefit from investments in steam savings, on the other hand, is nothing without the opportunity to, for example, use it to produce electricity. A number of constraints in the optimization model are therefore needed to model these mass and energy balances of the plant, and a deep understanding of process integration is needed to understand the relations between the measures in order to formulate the constraints correctly. While we still aim at optimizing the timing of decisions with regard to uncertainty, we are also faced with the non-trivial problem of deciding on which combination of energy efficiency measures that should be realized, out of a number of possible energy savings and ways to increase energy exports or decrease energy imports.

\section{Methodology}

\subsection{General approach}

We propose a methodology in five steps for decision-making about process integration investments when uncertainty is taken into account. A stochastic programming model has been developed, and the methodology describes the steps needed to achieve the necessary input as well as some proposals for analyzing the results.

The model is based on an energy systems analysis of the plant considered. Figure 1 illustrates the idea of such an analysis. First, a number of heat-saving measures are identified. These can be an improved heat exchanger network, new and more efficient equipment, or technology that replaces steam by excess heat of lower temperatures. The realizations of the identified opportunities for heat savings provide, primarily, a possibility to decrease fuel imports at the plant. In the case of a plant with combined heat and power production, this will also lead to decreased electricity production. A decrease of the fuel inputs might also affect the excess heat available for district heating. The benefit of decreasing the fuel costs thus has to be weighed against the investment costs for both the heat-saving measures and any decreases in power and district heating deliveries. The pulp industry is a special case, because many chemical pulp mills are selfsufficient in energy supply and moreover, the fuel is biomass, which primarily is a raw 
material for the process. Hence, for such mills heat savings, typically steam savings, will lead to a heat surplus at the mill, which enables increased electricity production, or even exports of biofuel.

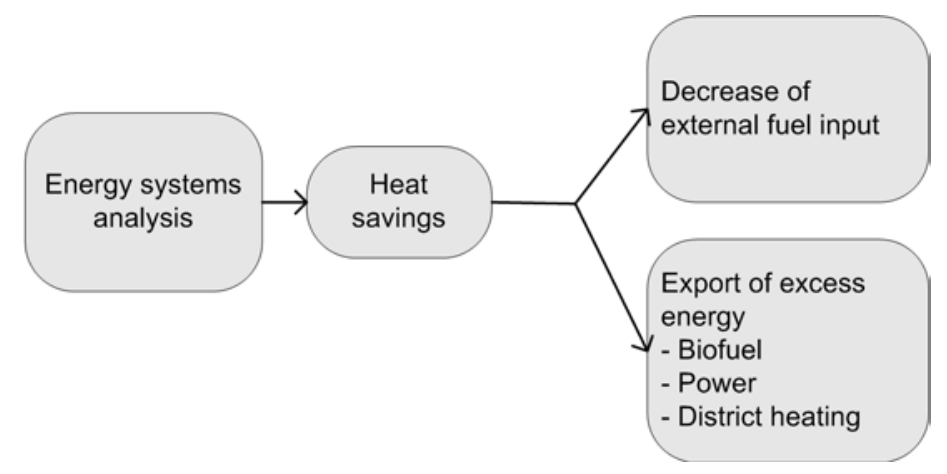

Figure 1: Energy systems analysis for identification of possible energy efficiency measures at an industrial plant.

Most of these strategies to improve the energy efficiency of the plant will lead to a reduction of $\mathrm{CO}_{2}$ emissions, for example by reducing the use of fossil fuels. Although biomass is generally assumed to be $\mathrm{CO}_{2}$-neutral, the reduction of biofuel use or of electricity production from biofuels will also lead to $\mathrm{CO}_{2}$ emissions reductions. The explanation is that, since biomass should be considered a limited fuel resource, a reduced usage at a particular plant enables the substitution of fossil fuels elsewhere, thereby reducing overall emissions. ${ }^{1}$

Not all identified measures for heat savings can be combined, however, and it is not obvious which is the optimal way to benefit from the improved energy efficiency. Hence, we need a way to decide which measures yield the highest profit, a task which is complicated by the uncertainty of future electricity and fuel prices.

The model is developed to be as general as possible, without limiting its usage to a specific industry, or a specific set of energy efficiency measures. The model is a multistage mixed-binary linear stochastic programming model. It contains a number of binary variables, which typically denote whether investments are made or not. It is assumed that investment decisions are made 'here-and-now', which means that they are decided on and implemented before uncertainties are resolved and any price changes occur. After the uncertainties have been resolved, new 'here-and-now' decisions might be made, for example on additional investments, before any further price changes occur. An article presenting a more detailed description of the mathematical model will be published (Svensson et al., 2008a).

The solution to the optimization model will be an optimal investment plan for strategic investments with respect to the NPV based on the information about the future that is available today. This means that, although results show that investments should be made perhaps ten years from now, decisions about those investments should not be made now. By then, not only will the true outcome of the uncertain parameters up to that point

\footnotetext{
${ }^{1}$ When the plant operates under a cap-and-trade emission trading scheme, there will be no $\mathrm{CO}_{2}$ emissions reductions in a short-term perspective. In a long-term perspective, however, energy efficiency improvements can be said to open up the possibility to decrease the cap, and thereby in the long run make it possible to achieve increased $\mathrm{CO}_{2}$ emissions reductions.
} 
be known, but also the knowledge of the future beyond that point will have increased. This should be taken advantage of by a renewed optimization of the investments, taking into account the changes and outcomes of the parameters that have occurred.

The main steps of the methodology are:

1. Identify the opportunities for energy efficiency investments.

2. Define the constraints on and effects of combining measures.

3. Gather and compute input data.

4. Develop a scenario model.

5. Solve the model and analyze the results.

Each of these steps is described in more detail in the following subsections.

\subsection{Step 1 - Identify the opportunities for energy efficiency investments}

The first step is to identify potential energy efficiency measures at the analyzed plant. This is done using process integration methods such as pinch analysis (Kemp, 2007; Smith, 1995). The identified efficiency measures render energy savings that either result in a heat surplus at the plant that can be used, for example, to produce electricity, or open up the possibility of decreasing the use of external fuel. Some examples of such measures are listed below:

- Improved internal heat exchange for the reduction of heating and cooling utilities.

- More energy-efficient equipment, e.g., efficient drying or separation processes.

- More efficiently integrated units, e.g., use of excess heat for distillation, etc.

- Heat pumps for increased heat recovery.

- A wide range of more sector-specific energy cost-saving measures.

In this step, the aim is to identify all relevant opportunities and to characterize these with respect to their associated investment costs and resulting energy savings. These opportunities are regarded as fixed, that is, with a fixed cost and a fixed energy saving. Measures that can be taken to different degrees of energy efficiency are included as separate alternatives, for which a successively higher investment cost yields successively higher energy savings.

As a consequence of the characterization of possible energy savings, it will become clear whether there exist more than one energy form or energy quality. These energy qualities, which might be, for example, different steam pressure levels, should also be noted in this step. Other than steam, energy savings might be in the form of hot water, heat in product streams, fuel savings, etc.

The implementation of investments in energy efficiency offers many possibilities to generate an income. There can be several possibilities to choose from (depending on the type of industry, situation, etc.). Following in the next paragraph, some possible opportunities to decrease the energy costs at a plant are presented in general terms. (Not all of them may be of interest in every case.)

Increased electricity generation in combined heat and power (CHP) plants will lead to either decreased import or increased export of electricity. Both steam turbines and gas turbines may be of interest. In some sectors as, for example, the food industry or the pulp and paper industry, the gas turbines will be combined with biomass gasification. Decreased fuel demand will make it possible to either sell surplus fuel or decrease purchases. Depending on the marginal fuel use at the plant, this will lead to changes in 
biofuel or fossil fuel use. Fuel switching is another way of decreasing the energy costs. Heat integration with nearby communities or industries through district heating is one way to utilize excess heat that would otherwise constitute a cooling demand for the process. Finally, $\mathrm{CO}_{2}$ capture and sequestration may, although it is not really an investment in energy efficiency, be an effective way to reduce costs related to energy if $\mathrm{CO}_{2}$ charges become high.

Some of the mentioned measures are still under development and are associated with uncertainties regarding investment costs and availability. Identifying which possibilities should be included in the analysis is also an aim of the first step.

\subsection{Step 2 - Define the constraints on and effects of combining measures}

Constraints that express how different process integration measures can be combined constitute an important part of the model, and the mathematical and logical formulations of these constraints are specific for each case study. If all opportunities identified in Step 1 could be realized, the energy savings would in many cases be substantial. This is, however, typically not the case: some measures can be combined, some can be combined under certain conditions, and some cannot be combined at all.

An example of the complexity of combining energy efficiency measures is when there are several ways of using excess heat. The alternatives might be, for example, district heating, process integrated distillation, or heat pumping. Here, the amount of excess heat is a limiting factor, and the alternative uses mentioned above cannot be combined. Additionally, with improved heat exchange, the available excess heat is decreased, which changes the conditions for the alternatives previously mentioned. They may still be possible to realize, but the associated costs and resulting heat savings may change. Further, other measures may cause heat temperature levels to change.

It is not a trivial problem to analyze all opportunities for energy efficiency, and one has to keep in mind that if one investment is chosen, the energy-saving potential for others may change. Knowledge and good understanding of process integration, as well as process technology in general, is required.

\subsection{Step 3 - Gather and compute input data}

The basic constituents of the model are expressions that describe the investment costs, energy savings, and resulting output from utilizing the improved energy efficiency for different purposes. The resulting output is, for example, the amount of electricity generated in a turbine, the decreased fuel imports, or the amount of district heating delivered to a district heating network. The profit and the associated $\mathrm{CO}_{2}$ emissions decrease can then be computed as a function of the output (from the turbines, district heating, etc.) at different times.

A number of constraints describe the mass and energy balances at the plant. Energy can be transformed from higher to lower qualities and a balance is formulated for each quality. Consider, as an example, the case of steam savings at a pulp mill. The mass balances are, in this case, steam balances for each steam pressure level, with the opportunity to pass steam from higher to lower pressures. It might be, under certain circumstances, possible to increase the output without any heat savings. This is the case for new equipment (such as a new turbine), for which the output (the electricity generated) is increased compared to that of an old one, simply because it has a higher efficiency. 
The possible increase in energy exports or decrease in energy imports depends on the extent to which energy savings have been implemented as well as on the installed capacity or size of process equipment such as turbines. It is assumed that the investment costs of process equipment are given by nonlinear concave functions. Since we assume a mixed-integer linear programming model, these are automatically approximated as piecewise linear functions before the optimization is carried out.

In the mathematical model, the above relations are modelled in a very general way, without any need to adjust the expressions for each specific case. However, the necessary input data need to be specified. As mentioned above, the profit depends on the output, but also on future prices, which leads us to the next step of the model.

\subsection{Step 4 - Develop a scenario model}

Because of the high uncertainty in future energy prices, the mathematical model needs to be stochastic. Unlike a deterministic model where typically the expected values of the uncertain parameters are used as input, the probability distributions of these parameters are taken into account in a stochastic programming model. Then, the expected value of the objective function is maximized, which often can lead to substantially different solutions compared to those obtained from solving a deterministic model corresponding to an average scenario.

In this step of the methodology a scenario model is developed. The model contains the prices and $\mathrm{CO}_{2}$ emissions for a number of scenarios at different times. It is impossible in practice to completely characterize the set of possible future outcomes. Simplifications are required not only to obtain a simple model, but also because there is no way of knowing the 'true' probability function. The advantage of using many scenarios, to cover many future possibilities, has to be weighed against the disadvantages of having to estimate the probability for each of the scenarios to occur and ending up with a very large-size optimization model.

Electricity and fuel prices are strongly correlated. Hence, we propose the use of a limited number of consistent energy market parameter sets, or building blocks, which can be used to build a scenario tree. These building blocks could (depending on the purpose of the study) represent, for example, a successively more ambitious emissions reduction target. By combining these blocks in different ways, a number of possible development paths towards the future are constructed. The probability of each of these paths should then be estimated, hence the need for simplification and a limited number of such paths.

In the process of constructing the development paths it becomes obvious that the time parameters need to be decided on in this step. The parameters of interest are the time intervals for which the building blocks are assumed to be valid, the economic lifetime of the investments, and the total time span analyzed. Although nothing in the mathematical model prevents these parameters from being chosen freely, some general aspects should be considered. It should be noted that the total time interval should not be longer than the economic lifetime of the investments. The economic lifetime has to be sufficient in order to analyze the timing of investments for a reasonable period of time. For many decisionmakers in industry, an economic lifetime of 30 years might seem too long, but in this kind of analysis it should be appropriate. It is important, though, as in any other economic evaluation, that the economic lifetime chosen should not be longer than the expected technical lifetime. It is of course also possible to choose a shorter lifetime, but it will set a limit on how far in the future the investment plan will be valid. While discussing the 
choice of the economic lifetime, it is also appropriate to decide on the discount rate to use.

For investments made close to the end of the analyzed time period, a residual value is calculated accounting for cash flows that would occur after the analyzed time period. Calculating those cash flows would require detailed data and information regarding the management and operation of equipment after its presupposed economic lifetime. It is difficult to know what decisions will be taken regarding equipment that is no longer operating efficiently. If it continues to be in operation, the maintenance costs and shutdown times might increase. Alternatively, it may be shut down permanently, possibly yielding a scrap value, but then new investments have to be made, either for a similar piece of equipment, or for an alternative process solution. Hence, simplifications are necessary. In the proposed model, the residual value is chosen to even out the annualised investments for the missing years. This should be a reasonable approach, since the cash flows estimated in this way will neither increase nor decrease the net present value of the investments.

At present, the model can handle uncertainties in prices and $\mathrm{CO}_{2}$ emissions. It would also be of interest to incorporate uncertainties in investment costs and availability of new technologies. This would directly imply the need for even more scenarios, and a model for the relations between the different uncertain parameters would then be necessary. To incorporate other sources of uncertainty is not the aim of this study, but will be an interesting continuation.

\subsection{Step 5 - Solve the model and analyze the results}

Under the assumptions made here, the resulting optimization model will be a multistage mixed-binary linear programming model. There are both commercial and free software available for formulating and solving such models. The default objective of the model is the net present value (NPV), but associated $\mathrm{CO}_{2}$ emissions reductions can also be studied.

A number of customizations can be done in order to analyze the results. The total number of investment occasions can be limited. Alternatively, the total investment for each investment occasion may be limited. If the limit is not reached, money can be saved until the next period. A limit on $\mathrm{CO}_{2}$ emissions can be set, thus enabling the possibility of analyzing the trade-off between maximizing the NPV and minimizing the $\mathrm{CO}_{2}$ emissions. A way to handle risk more explicitly is to set a limit for the worst-case scenario NPV.

It should also be noted that it is possible to employ an iterative procedure for the five steps. Solutions that are not suitable, for one reason or another, may be excluded by the introduction of new constraints in order to avoid only the unwanted investments or operating conditions. These constraints could of course have been introduced already in Step 2. The advantage of not introducing all constraints at once is that more knowledge, particularly about the advantages with a flexible system, can be obtained. This is an opportunity for an interactive procedure that will increase the insights regarding the system characteristics.

The results can be analyzed in a variety of ways, some of which have already been mentioned. It is also recommended to vary the probability distribution of the development paths in order to investigate how important the estimate of the distribution is. This is a sensitivity analysis that can be used to check the stability of the solution. Furthermore, the economic parameters such as the economic lifetime and the discount rate should be varied in order to investigate the difference between a short-term perspective solution and a 
more strategic one. This might affect the developed scenario model, which then has to be changed accordingly.

For all analyses it is important to remember that the solution value (the NPV) might vary substantially for different sets of economic parameters, for different scenarios, etc., while the characteristics of the optimal solution regarding the optimal initial investment might still be the same.

\section{Summary and conclusions}

To summarize, this paper presents a methodology that has been developed for evaluating investments in energy efficiency measures with respect to their net present values and possibly also environmental objectives. The methodology considers explicitly the uncertainties of future energy prices and policies while, in addition, a number of constraints can be formulated to model the complex relations between different process integration opportunities. The aim of the methodology is to combine knowledge of process integration and stochastic programming in order to receive a better basis for investment decisions.

A step-by-step procedure is proposed, beginning with the identification of opportunities for, as well as constraints on, the implementation of energy efficiency measures. The following steps consider data collection for the energy balances and the technical and economic parameters, including the uncertain parameters. Finally, the model is solved and the results are analyzed. The methodology developed does not limit the kinds of analyses that can be carried out in this step, but some suggestions are provided in the description of the methodology. The usefulness of the methodology is justified by the fact that it is the first approach to apply a multistage stochastic programming approach for the optimization of these kinds of investments in process integration. The methodology is illustrated in Svensson et al. (2008b), where a case study using the described methodology for the evaluation of energy efficiency measures at a pulp mill is presented, see also Svensson et al. (2008a).

The methodology provides a framework for analyzing investment alternatives in which complex combinations of energy-efficiency measures are possible to implement. The method is not limited to comparing a few discrete alternatives as is often the case in a real options approach. Uncertainties and the time-dependence of parameters such as energy prices and policies are explicitly incorporated in the optimization model. This takes the handling of uncertainties far beyond the way they are handled in a traditional sensitivity analysis, thereby enabling the identification of robust solutions.

\section{Acknowledgements}

Financial support by the Swedish Energy Agency (the contributions by the first three authors of the paper) and the Swedish Foundation for Strategic Research through the Gothenburg Mathematical Modelling Centre (the contribution by Michael Patriksson) is gratefully acknowledged. 


\section{References}

Birge, J.R., Rosa, C.H., 1996. Incorporating investment uncertainty into greenhouse policy models. Energy Journal, 17, 79-90.

Birge, J.R., Louveaux, F., 1997. Introduction to Stochastic Programming. SpringerVerlag, New York.

Blyth, W., Bradley, R., Bunn, D., Clarke, C., Wilson, T., Yang, M., 2007. Investment risks under uncertain climate change policy. Energy Policy, 35, 5766-5773.

Diederen, P., van Tongeren, F., van der Veen, H., 2003. Returns on investments in energy-saving technologies under energy price uncertainty in Dutch greenhouse horticulture. Environmental and Resource Economics, 24, 379-394.

Dixit, A.K., Pindyck, R.S., 1994. Investment under Uncertainty. Princeton University Press, Princeton.

FRAM, 2005. FRAM Final report: Model mills. STFI, Stockholm, Sweden.

Fuss, S., Szolgayova, J., Obersteiner, M., Gusti, M., 2008. Investment under market and climate policy uncertainty. Applied Energy, 85, 708-721.

IEA, 2007. Tracking Industrial Energy Efficiency and $\mathrm{CO}_{2}$ Emissions. OECD/IEA, Paris, France.

Kall, P., Mayer, J., 2005. Stochastic Linear Programming: Models, Theory and Computation. Springer, New York.

KAM, 2003. Eco-Cyclic Pulp Mill Research Program, Final report 1996-2002, A100. STFI, Stockholm.

Kemp, I.C., 2007. Pinch Analysis and Process Integration: A User Guide on Process Integration for the Efficient Use of Energy. Butterworth-Heinemann, Oxford.

Laurikka, H., 2006. Option value of gasification technology within an emissions trading scheme. Energy Policy, 34, 3916-3928.

Louveaux, F., Schultz, R., 2003. Stochastic integer programming, in: Ruszczyński, A., Shapiro, A. (Eds.), Stochastic Programming, Vol. 10 of Handbooks in Operations Research and Management Science. Elsevier, Amsterdam, pp. 213-266.

Ruszczyński, A., Shapiro, A. (Eds.), 2003. Stochastic Programming, Vol. 10 of Lenstra, J.K., Nemhauser, G.L., Handbooks in Operations Research and Management Science. Elsevier, Amsterdam.

Saltelli, A., Tarantola, S., Campolongo, F., Ratto, M., 2004. Sensitivity Analysis in Practice: A Guide to Assessing Scientific Models. John Wiley \& Sons, West Sussex.

Sen, S., 2005. Algorithms for stochastic mixed-integer programming models, in: Aardal, I., Nemhauser, G.L., Weismantel, R. (Eds.), Discrete Optimization. Elsevier, Amsterdam, pp. 515-558.

Smith, R., 1995. Chemical Process Design. McGraw-Hill, Inc, New York.

Svensson, E., Strömberg, A.-B., Patriksson, M., 2008a. A scenario-based stochastic programming model for the optimization of process integration opportunities in a 
pulp mill. Preprint ISSN 1652-9715, No 2008:29. Mathematical Sciences at Chalmers University of Technology and University of Gothenburg, Göteborg, Sweden.

Svensson, E., Berntsson, T., Strömberg, A.-B., 2008b. Benefits of using an optimization methodology for identifying robust process integration investments under uncertainty - A pulp mill example. Submitted to Energy Policy.

Wallace, S.W., 2000. Decision making under uncertainty: is sensitivity analysis of any use? Operations Research, 48, 20-25.

Wickart, M., Madlener, R., 2007. Optimal technology choice and investment timing: A stochastic model of industrial cogeneration vs. heat-only production. Energy Economics, 29, 934-952.

Yang, M., Blyth, W., Bradley, R., Bunn, D., Clarke, C., Wilson, T., 2008. Evaluating the power investment options with uncertainty in climate policy. Energy Economics, 30, 1933-1950.

Ådahl, A., Harvey, S., 2007. Energy efficiency investments in kraft pulp mills given uncertain climate policy. International Journal of Energy Research, 31, 486-505. 